\title{
Two cases of atypical foramen magnum meningioma presenting as rotatory paralysis
}

\begin{abstract}
Meningiomas are the most common benign intracranial neoplasms accounting for 13 to $19 \%$ of all intracranial tumors. Only 1.8 to $3.2 \%$ arise at the foramen magnum (FM).However foramen magnum meningioma are usually typical meningioma .Atypical meningioma in foramen magnum is very rare. Only one case report was found so far in literature. As they produce delayed symptoms so their development at the craniocervical junction makes clinical diagnosis complex and often leads to a long interval between onset of symptoms and diagnosis. We report 2 cases of atypical meningiomas of foramen magnum presenting as rotatory paralysis. We will here discuss clinical as well as operative course of this case in a nut shell
\end{abstract}

Keywords: atypical meningioma, foramen magnum, craniospinal junction, far lateral approach, rotatory paralysis
Volume 9 Issue 4 - 2019

Moududul Haque, Nwoshin Jahan, Shahnawaz Bari, KM Tarekul Islam, Shamsul Alam, ATM Mosharef Hossain, Ashifur Rahman Bijou, Rajib Bhattacharya, Bipin chaurasia

Department of Neurosurgery Bangabandhu Sheikh Mujib Medical University, Bangladesh

Correspondence: : Bipin chaurasia, Resident, Department of Neurosurgery Bangabandhu Sheikh Mujib Medical University, shahbagh, Dhaka, Bangladesh, Email trozexa@gmail.com

Received: June 20, 2019 | Published: July 01, 2019

\section{Introduction}

Meningioma is extra axial tumor originate from arachnoid cap cells found in the arachnoid layer of meninges. They have an annual incidence of six per 100,000 people. An estimated $1-2 \%$ of the population has incidental asymptomatic meningiomas. And in autopsy studies $8 \%$ of these are multiple.$^{1-3}$ It occur more commonly in female. Meningiomas account for $20 \%$ of all intracranial tumors in males and $38 \%$ in females. Overall brain tumor meningioma account for 13 to $19 \%$. Spinal meningioma account for $12 \%$ of all meningioma and $25 \%$ to $45 \%$ of all spinal tumor. ${ }^{4}$ According to WHO classification meningiomas are designated as benign (grade I) account for $90 \%$, atypical (grade II) $6 \%$ and anaplastic/malignant (grade III) $2 \%$ of cases. Atypical meningiomas deviate from their benign counterparts by the presence of increased mitotic activity or three or more of the following changes: increased cellularity, small cell formation, prominent nucleoli, sheet like growth, and areas of spontaneous necrosis. Foramen magnum meningiomas (FMMs) are a rare entity. Only $1.8-3.2 \%$ of all meningiomas are located at foramen magnum (FM).

\section{Case report}

\section{Case no I}

A 25years female admitted in our department of Neurosurgery with the complaints of neck pain for 1 year, progressive weakness of all limbs in the form of rotating paralysis starting with right upper limb followed by right lower limb, left lower limb \& left upper limb for 8months with bowel bladder disturbance in the form of urgency $\&$ frequency, on neurological examination patient was found to be quadri-paretic having muscle power Medical Research Council (MRC) grade 4 on right side \& 3 on left side rendering her gait to be hemiplegic and walk with support. All her deep tendon reflexes were exaggerated with bilateral planter extensor. Ankle clonus was present bilaterally. She did not have any hypo or hyper pigmented patch in any part of the body. Magnetic resonance imaging (MRI) of brain \& cervical spine showed an intra-dural extra-medullary lesion measuring about $3.5 \times 2.7 \mathrm{~cm}$ at foramen magnum extending both up and down.it was placed more on right side crossed the midline and almost touched the opposite margin of the foramen magnum. Another intra-dural extra-medullary lesion was also noticed at the level of $\mathrm{C} 4 / 5$ region (Figure 1) \& (Figure 2). There was no other lesion on further cranial \& spinal screening MRI.

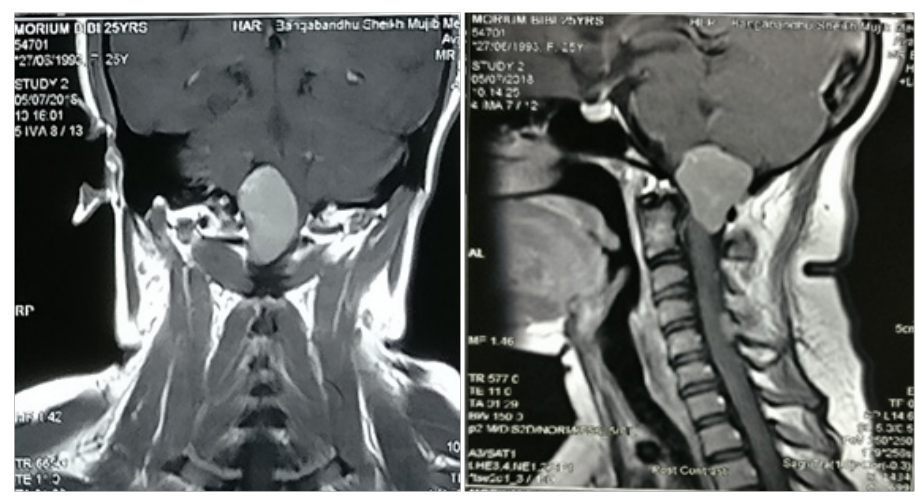

Figure I MRI revealed right FM meningioma with $3.5 \times 2.7 \mathrm{~cm}$

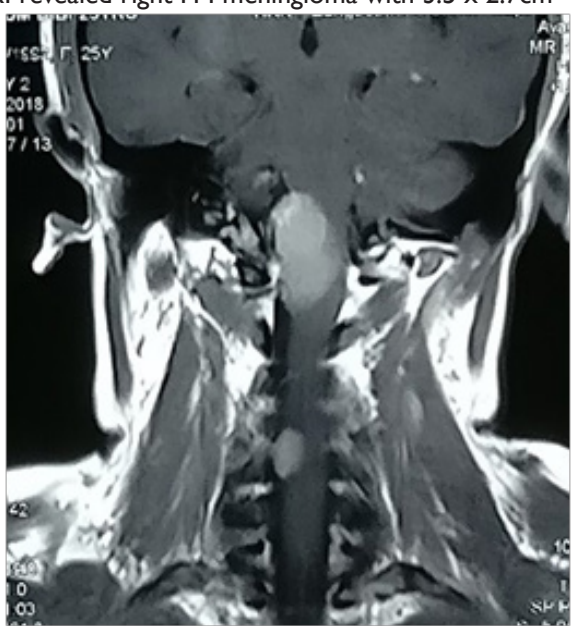




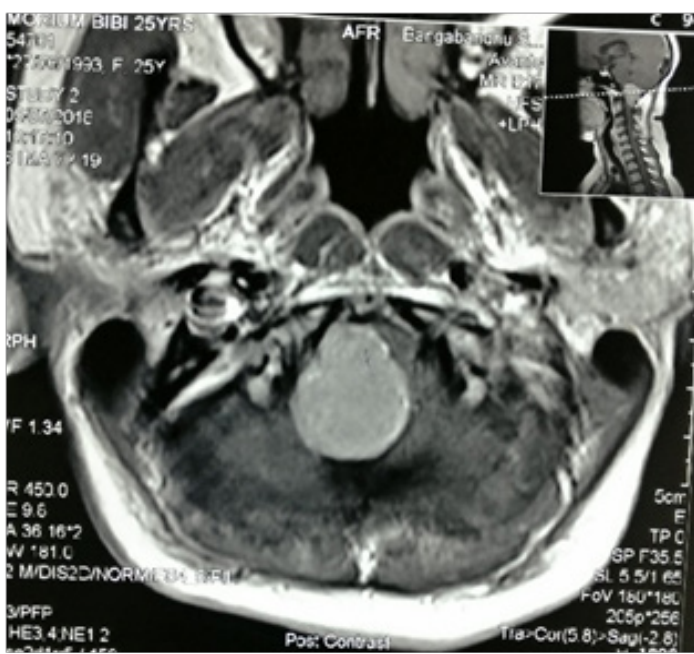

Figure 2 MRI revealed duel pathology at right FM \& at C4/5 level.

Through far lateral approach the lesion was removed totally in a piecemeal fashion .Simpson grade I was achieved. Histopathology revealed atypical meningioma WHO grade II. She started to improve gradually and was discharged to home. After 3 months of follow up she showed marked improvement of power in her all limbs. MRC grade improves to 5 in all limbs which enable her to walk independently. And regarding the another lesion, as it is asymptomatic \& currently patient $\&$ her relative not agree to go for another surgery so our plan is regular follow up of the patient.

\section{Case no 2}

A 34years male admitted in our Department of Neurosurgery with the complaints of neck pain for 2 year, progressive weakness of all limbs in the form of rotating paralysis starting with left upper limb followed by left lower limb, right lower limb \& right upper limb for 15 months without bowel bladder disturbance. On neurological examinations patients was found to be quadriparetic having muscle power MRC grade 3 on both side rendering her gait to be hemiplegic and walk with support. All her deep tendon reflexes were exaggerated. Hoffman sign was positive bilaterally. Planters were bilaterally extensor. Ankle clonus was present bilaterally. He did not have any hypo or hyper pigmented patch in any part of the body. MRI of brain \& cervical spine showed an intra-dural extra medullary lesion measuring about $5 \times 3.5 \mathrm{~cm}$ at foramen magnum extending both up and down situated posteriorly more on left side (Figures 4A-C). There was no other lesion on further cranial \& spinal screening MRI.

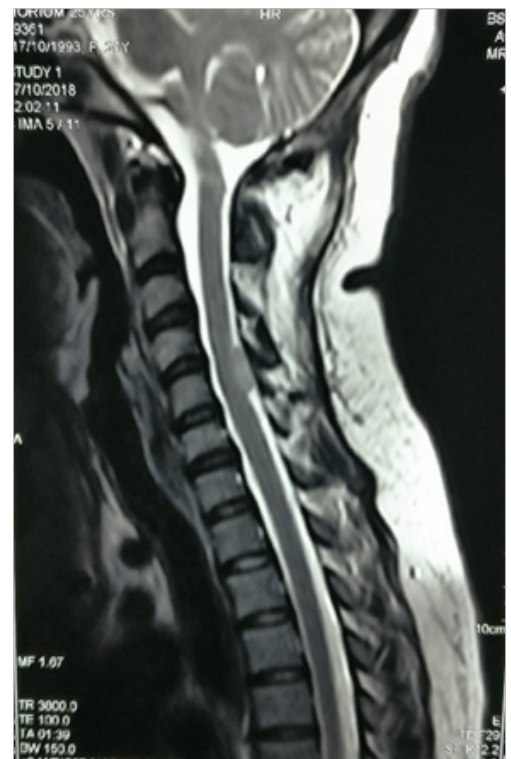

(A)

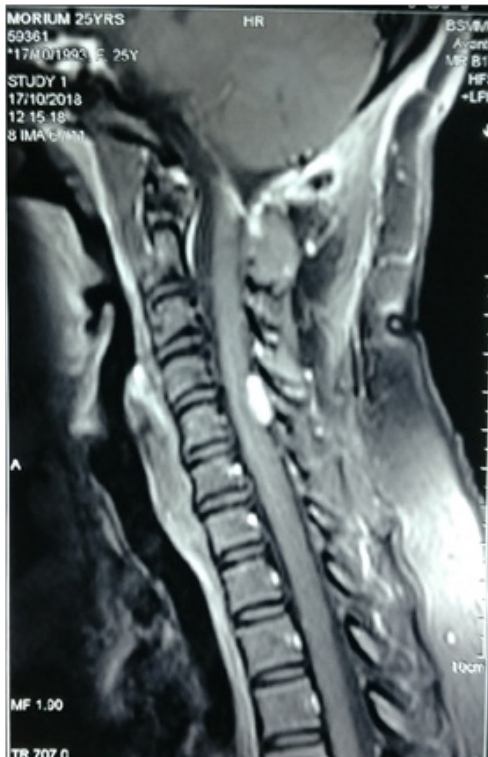

(B)

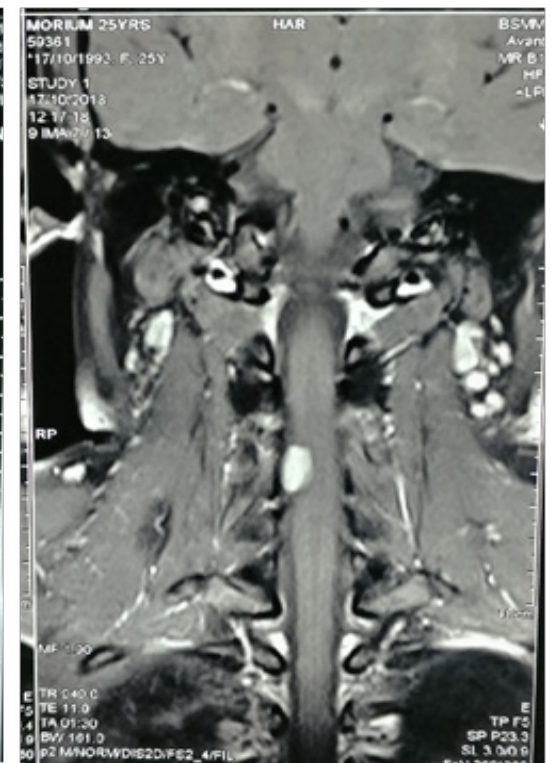

(C)

Figure 3 (A.T2 sagittal, B \& C are contrast sagittal and coronal respectively): post-operative image showing there is no residual tumor at FM.

After proper evaluation he underwent posterior midline sub occipital craniectomy with removal of $\mathrm{C} 1$ arch. Gross total removal of tumor was done with Simpson grade I. Tumor was firm in consistency, fibrous, non suckable, moderately vascular. There was pial invasion at the lower part of the medulla. Left sided PICA was severely attached with the tumor which was carefully separated. Dural attachment of tumor was found and involved dura was removed. Duroplasty was done with artificial dura. Post-operative recovery was uneventful. Tissue was sent for histopathology which reveals atypical meningioma WHO grade II. After surgery patients symptoms were improved gradually at 3 months of follow up. He showed marked improvement of power in his all limbs. And he can walk without any support. Post-operative MRI after 3 months show no residual tumors (Figures 5A-D).

\section{Discussion}

The pathologic entity of FMM was first described in 1872 . The first publication describing surgical removal was in $1922 .{ }^{5} \mathrm{~A}$ meningioma is considered to arise from the foramen magnum if the origin of the tumor is in the region bounded anteriorly by the lower third of the clivus and the upper edge of the body of $\mathrm{C} 2$, laterally by the jugular tubercles and the upper aspect of the $\mathrm{C} 1$ laminas, posteriorly by the anterior edge of the squamous occipital bone and C2 spinous process. The large majority $(90 \%)$ of these tumors are located ventrally or ventrolaterally. FMMs are typically slow growing with an indolent course, but when they become symptomatic, they most commonly present with quadriparesis, sensory abnormalities, 
ataxia, and dysfunction of cranial nerves (CN) IX and $\mathrm{X} .{ }^{6}$ The classic presentation of weakness associated with a FMM is initial weakness in the ipsilateral arm. Progression then occurs in the ipsilateral leg, the contralateral leg, and finally the contralateral arm. With compression at the Craniocervical junction, patients may also have downbeat nystagmus on physical examination. ${ }^{7}$ Other signs that may be present include wasting of the sternocleidomastoid, trapezius, or intrinsic muscles of the hand. Our both patients had the classical presentation of rotatory paralysis of the limbs; both of them developed their symptoms over a period of 1year. In one case symptoms start from right upper limb, this was most likely because of the involvement of the tumor predominantly on the right side with bowel bladder involvement. She did not have any other manifestation involving the sensory system or the cranial nerve. In another case symptoms start from left upper limb followed by left lower limb, right lower limb and right upper limb without bowel bladder and sensory of cranial nerve involvement .FMMs are strictly intradural. Ten percent have an extradural extension and a few may be entirely extradural .Based on
MRI examination, according to the insertion on the dura FMMs can be classified as anterior if origin is on both sides of the anterior midline, lateral if insertion is between the midline and the dentate ligament and postero-lateral if insertion is posterior to the dentate ligament. Anterior FMMs displace the neuroaxis posteriorly, lateral displace the neuroaxis postero-laterally and postero-lateral displace the neuroaxis ventrolaterally. ${ }^{8,9}$ Extradural meningiomas at the foramen magnum are less common than intradural tumors. Complete resection is more challenging due to their invasive nature. ${ }^{9}$ If tumor origins below the vertebra artery(VA) cranial nerves are displaced cranially and if origins above the VA the cranial nerve goes in variable location \& meticulous dissection must be carried out to prevent injury.Many advocate vascular imaging (CTA, MRA, or conventional angiography) preoperatively to evaluate arterial feeders, venous drainage, and the extent of vascular involvement. In particular, defining the V3 and V4 segments of the vertebral artery along with the origin of the PICA will aid in operative planning and the avoidance of complications. ${ }^{9}$

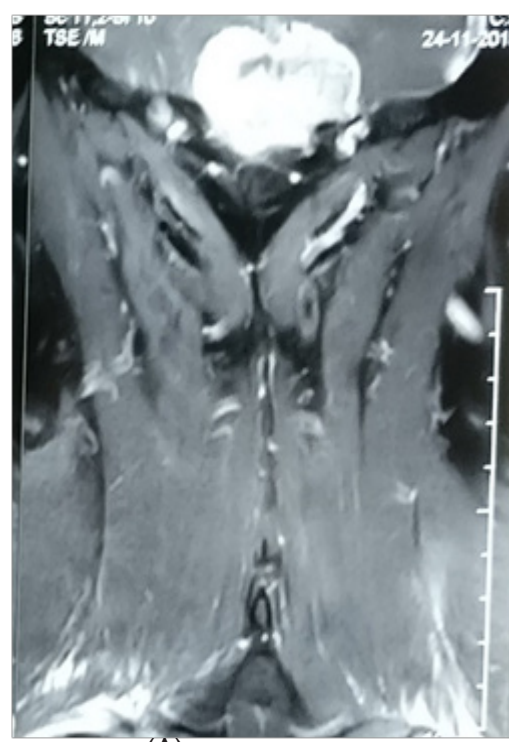

(A)

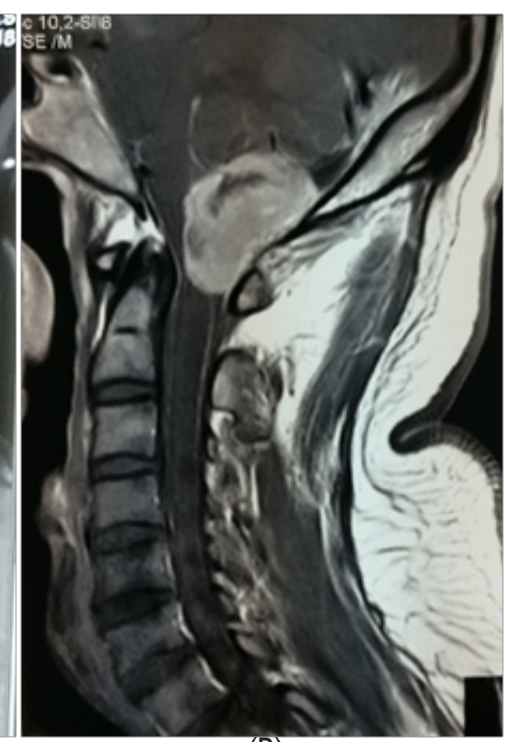

(B)

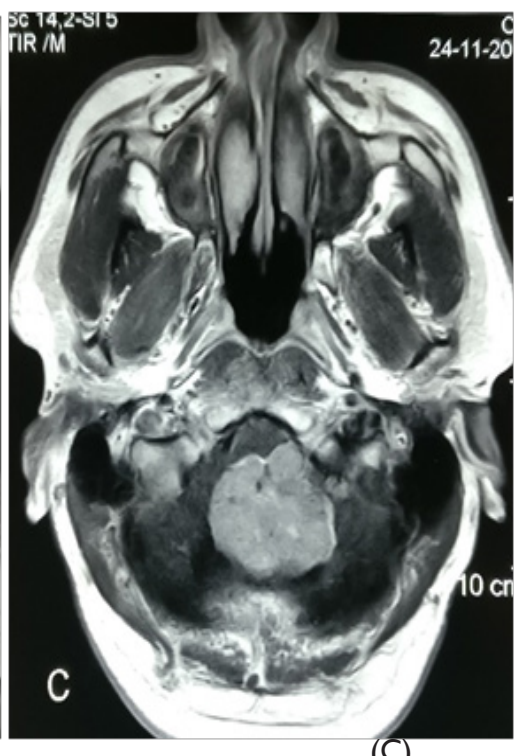

(C)

Figure $4 \mathrm{MRI}$ of brain \& cervical spine showed an intra-dural extra medullary lesion measuring about $5 \times 3.5 \mathrm{~cm}$ at foramen magnum extending both up and down situated posteriorly more on left side.

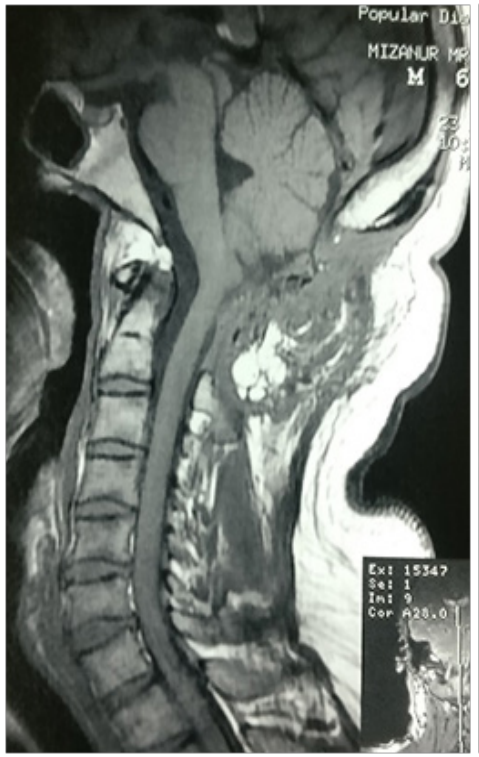

(A)

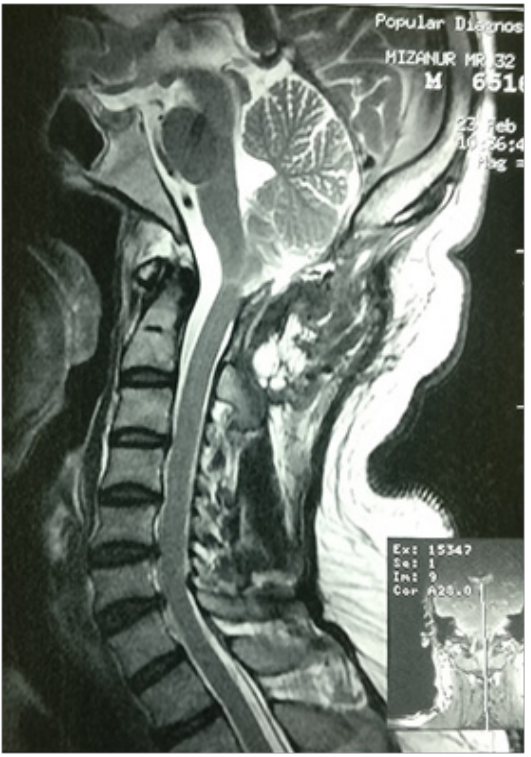

(B)

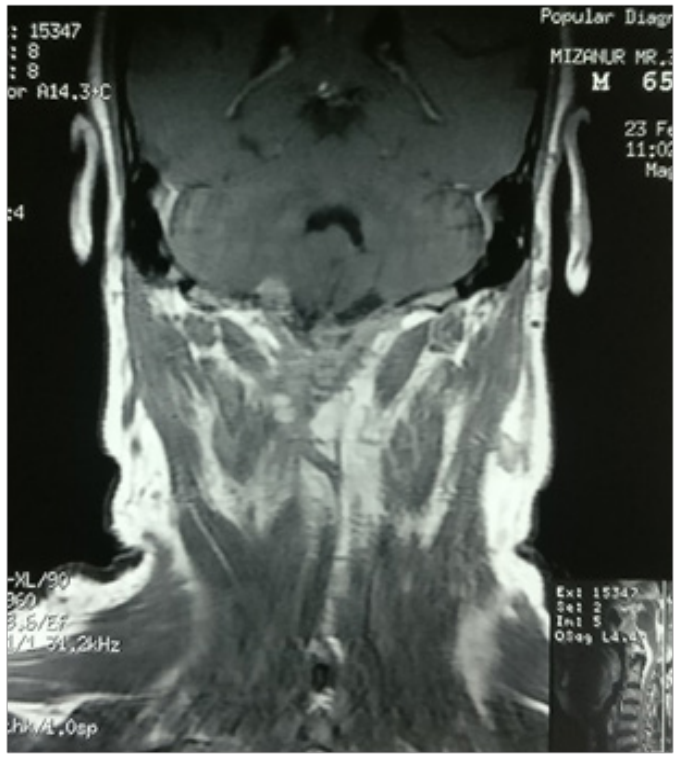

(C)

Citation: Haque M, Jahan N, Bari S, et al.Two cases of atypical foramen magnum meningioma presenting as rotatory paralysis. J Neurol Stroke. 2019;9(4):I80-I82. DOI: I0.15406/jnsk.2019.09.00373 


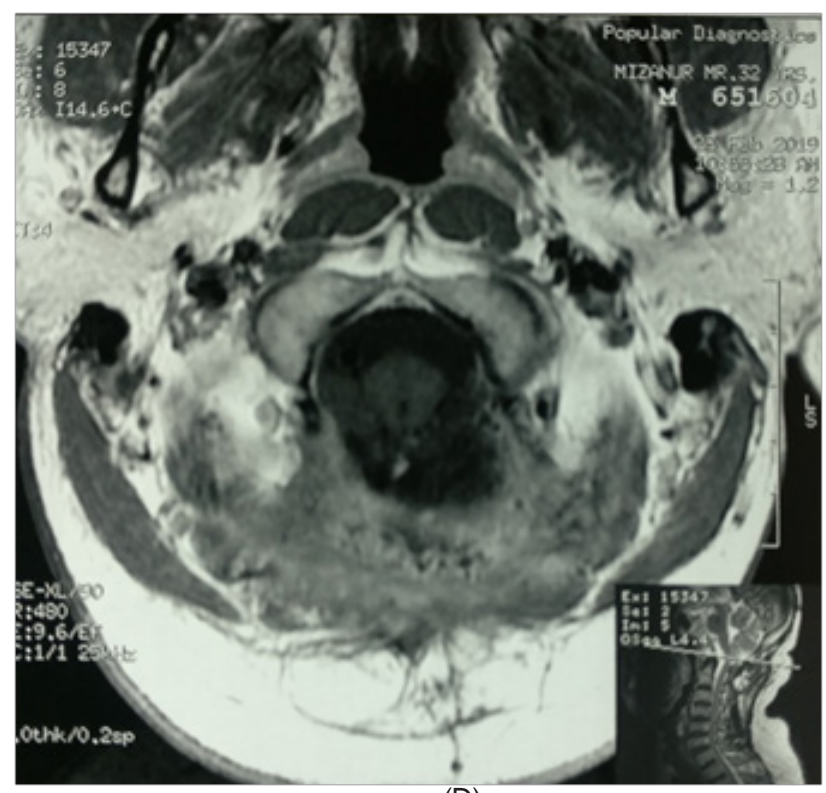

(D)

Figure 5 Post-operative MRI after 3 months show no residual tumors.

The incidence of atypical meningioma is $8 \%$ of all meningioma and the incidence is increasing day by day. ${ }^{10}$ Interestingly both of our case was atypical meningioma WHO grade II. From the study of Pamir et al. they operated total 22 cases of foramen magnum meningioma from January 1990 to 2003. Among those cases only one case were atypical meningioma. ${ }^{11}$ There is some controversy with the role of post operative radiation therapy in case of atypical meningioma. Moreover surgery with radiation in compare to surgery alone doesn't show any beneficial effect. ${ }^{10,12,13}$ However from literature we can say that radiation therapy has no role in preventing recurrence rate of atypical meningioma after gross total resection..$^{14,15}$

\section{Conclusion}

Foramen magnum meningiomas are not common disease. Whenever it occurs in this location it usually presents with typical features of rotatory paralysis. Surgical decompression of foramen magnum is blessing for patients as patients improve dramatically. Foramen Magnum meningiomas so far published in the literature are mostly found to be typical meningiomas. Atypical meningiomas of foramen magnum are very rare. And we should keep in mind of this pathology whenever histological differential diagnosis is concerned.

\section{Acknowledgments}

None.

\section{Conflicts of interest}

Author declares there are no conflicts of interest towards the article

\section{References}

1. Jurinovic P, Bulicic AR, Marcic M, et al. Foramen magnum meningioma: a case report and review of literature. Acta Inform Med. 2016;24(1):74.

2. Alam Shamsul, Bipin Kumar Chaurasia, Narendra Shalike, et al. Surgical management of clinoidal meningiomas: 10 cases analysis. Neuroimmunology and Neuroinflammation. 2018;5:21.

3. Chaurasia B, Hossain M, Barua KK, et al. Meningiomatosis in nonneurofibromatosis patient: how we dealt with 8 tumors. $J$ Neurol Stroke. 2018;8(6):307-310.

4. Nakamura M, Tsuji O, Fujiyoshi K, Hosogane N, Watanabe K, Tsuji T, Ishii K, Toyama Y, Chiba K, Matsumoto M. Long-term surgical outcomes of spinal meningiomas. Spine. 2012;37(10):E617-E623.

5. Flores BC, Boudreaux BP, Klinger DR, et al. The far-lateral approach for foramen magnum meningiomas. Neurosurg Focus. 2013;35(6):E12.

6. Talacchi A, Biroli A, Soda C, et al. Surgical management of ventral and ventrolateral foramen magnum meningiomas: report on a 64-case series and review of the literature. Neurosurg Rev. 2012;35(3):359-367.

7. Boulton MR, Cusimano MD. Foramen magnum meningiomas: concepts, classifications, and nuances. Neurosurg Focus. 2003;14(6):e10.

8. George B, Lot G, Boissonnet H. Meningioma of the foramen magnum: a series of 40 cases. Surg Neurol. 1997;47(4):371-379.

9. Bruneau M, George B. Foramen magnum meningiomas: detailed surgical approaches and technical aspects at Lariboisière Hospital and review of the literature. Neurosurg Rev. 2008;31(1):19-32.

10. Pereira BJ, de Almeida AN, Paiva WS, et al. Impact of radiotherapy in atypical meningioma recurrence: literature review. Neurosurg Rev. 2018;19:1-7.

11. Pamir MN, Kılıç T, Özduman K, Türe U. Experience of a single institution treating foramen magnum meningiomas. $J$ Clin Neurosci. 2004;11(8):863-867.

12. Champeaux C, Dunn L. World Health Organization grade II meningiomas. Acta Neurochir (Wien). 2016;158(5):921-929.

13. Endo T, Narisawa A, Ali HS, Murakami K, Watanabe T, Watanabe M, Jokura H, Endo H, Fujimura M, Sonoda Y, Tominaga T. A study of prognostic factors in 45 cases of atypical meningioma. Acta Neurochir (Wien). 2016;158(9):1661-1667.

14. Claus EB, Bondy ML, Schildkraut JM, et al. Epidemiology of intracranial meningioma. Neurosurgery. 2005;57(6):1088-1095.

15. Richardson AM, Au K, Morcos J. Foramen Magnum Meningiomas. In Skull Base Surgery of the Posterior Fossa 2018. Springer, Cham. 2018. p. $135-143$. 